\title{
Subjective evaluations of the sound field in a piano practice room with reflective sounds added using electro-acoustic technology
}

\author{
Takashi Fujita and Shigeo Ando \\ Yamaha Corporation, \\ 10-1, Nakazawa-cho, Hamamatsu-shi, 430-8650 Japan
}

(Received 19 October 1998)

\begin{abstract}
Subjective evaluations of a sound field actively controlled by adding reflective sounds in a piano practice room were made in terms of "Reverberance", "Spatial impression", "Home practice" and "Hall practice". The evaluations made by actually playing the piano and made when listening binaurally through headphones were compared. The evaluations were almost the same, but when actually playing the piano, the piano player's preference for reverberance strongly influenced the evaluations and especially influenced the evaluation of "Home practice". This suggests that it may be neccesary to take into account the sound absorption of a room as well as the piano player's preference for reverberance in the sound field actively controlled by adding reflective sounds using electroacoustic technology.
\end{abstract}

Keywords : Active sound field control, Subjective evaluation, Piano practice room, Sound absorption, Howling margin

PACS number: 43.55 . Hy

\section{INTRODUCTION}

The authors examined the relationships of physical values such the amount of added reflective sounds and/or sound absorption of a room to subjective evaluations of active sound field control in a piano practice room. ${ }^{1,2)}$

A previous report ${ }^{2)}$ clarified the relationships of the effects of added reflective sounds to the howling margin and the influence of sound absorption on added reflective sounds. In a subjective evaluation subjects evaluated the sound stimuli of piano playing sounds by listening binaurally through headphones. These sounds were dummy-head-recorded under various sound field conditions. The evaluations of the sound field were made in terms of "Reverberance", "Spatial impression", "Home practice" and "Hall practice". It was necessary to confirm that the same evaluations would be obtained when the player actually played the piano in a piano practice room.
This paper describes subjective evaluations done in a real sound field and compares the results with those obtained by listening binaurally through headphones.

\section{CHANGES IN SOUND ABSORPTION AND THE ACTIVE SOUND FIELD CONTROL SYSTEM IN A PIANO PRACTICE ROOM}

The experiment was done in a sound-proofed piano practice room. The dimensions of the room were $3.3 \mathrm{~m} \times 4.2 \mathrm{~m} \times 2.2 \mathrm{~m}$. The plan of the room is shown in Fig. 1. The room's sound absorption was changed by setting absorbing fiberglass boards or reflective plywood boards against the walls.

Figure 2 shows the values of the frequency characteristics of the reverberation time (RT) and the average absorption coefficients $(\bar{\alpha})$ for three types of sound absorption. The average values of $\bar{\alpha}$ from $250 \mathrm{~Hz}$ to $2 \mathrm{kHz}$ for these three types resulted in 0.29 , 0.36 , and 0.47 which are roughly equal to $0.3,0.35$, 


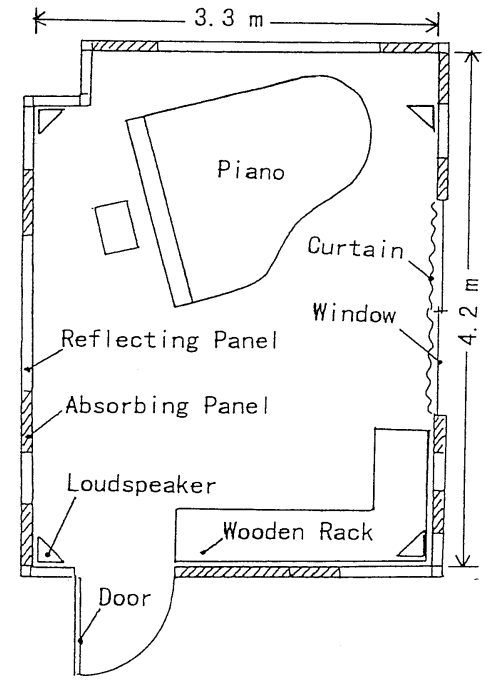

Fig. 1 Plan of the piano practice room used for the experiment. Floor $=$ wooden flooring; Ceiling = absorbing panel; Height $=2.2 \mathrm{~m}$. Interior wall configuration is sound absorption condition [1].

0.5 in previous experiment. ${ }^{2}$ Here, the value 0.29 corresponds to a generally approved sound field treated by architectural means ${ }^{3)}$ (this condition is "sound absorption condition [1]," which is indicated by the symbol in the figures). The value 0.36 corresponds to one of the effective conditions for the active sound field control in the previous experiment (this condition is "sound absorption condition [2]," which is indicated by the symbol $O$ in the figures). The value 0.47 corresponds to a condition which is highly absorptive (this condition is "sound absorption condition [3]," which is indicated by the symbol $\bullet$ in the figures).

A sound field reproduction system which synthesizes four channel reflections by using a DSP (digital signal processor) was used to add reflective sounds. ${ }^{1,2)}$ Figure 3 shows the block diagram of this system.

In order to add reflective sounds, first, the sound signal from the piano was collected by an omnidirectional boundary microphone placed on the floor of the room. Next, the sound signal was sent to the DSP which is composed of independent four channel FIR (finite impulse response) filters. In the DSP, the piano sound signal was convoluted with the four channel reflection data. After going through a four channel power amplifier, the
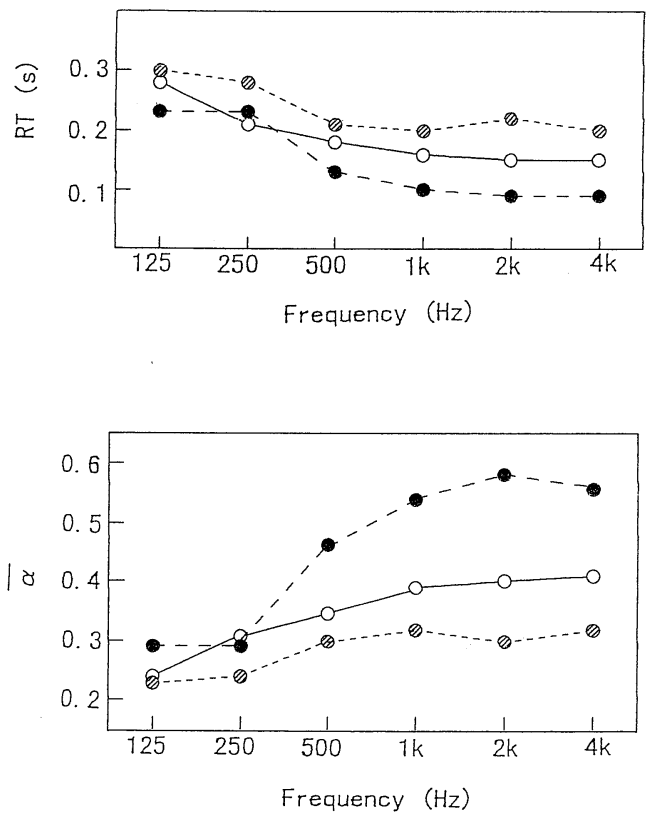

Fig. 2 Frequency characteristics of measured values of reverberation time (RT) and average absorption coefficient $(\bar{\alpha})$ in the piano practice room. sound absorption condition [1]; 0 , sound absorption condition [2]; $\bullet$, sound absorption condition [3].

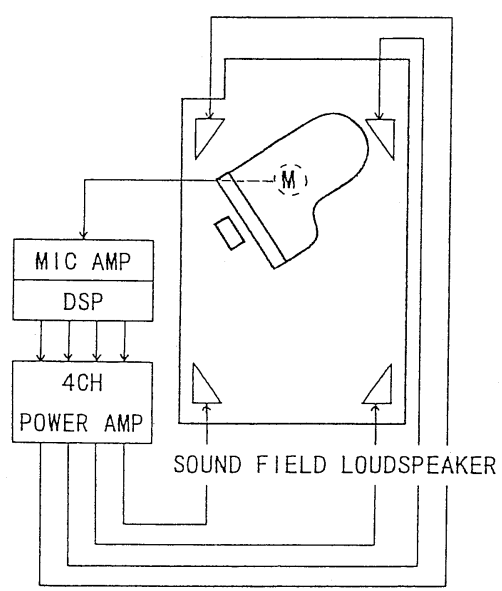

Fig. 3 Block diagram of the active sound field control system of the piano practice room.

convoluted four channel signals were reproduced through four loudspeakers set at four corners of the ceiling of the room and reflective sounds were added.

Data measured in the actual hall using a closely 
located four point microphone method ${ }^{4}($ shoebox type, volume $=15,000 \mathrm{~m}^{3}, \mathrm{RT}(500 \mathrm{~Hz})=2 \mathrm{~s}$, the same one as in the previous papers, ${ }^{1,2)}$ ) was utilized as the reflection data used here. The spatial information obtained using this microphone method is described as virtual sound sources composed of amplitudes, delay times, and directions of the reflective sounds. These virtual sound sources were projected onto the horizontal plane which corresponded to the plane of the four loudspeakers in the room. The virtual sound sources placed between two neighboring loudspeakers were reproduced by each loudspeaker's reflective sounds which have ratio of sound volumes and delay times. The localization of the sound sources in this case was almost good from the result of a listening test. ${ }^{5)}$

In the previous experiments, ${ }^{1)}$ it was confirmed that the degree of sound field effects on the added reflective sounds can be controlled by the howling margin (HM).

\section{SUBJECTIVE EVALUATION}

In this experiment, each subject played a piece of music selected by oneself optionally in the various sound field conditions. They evaluated the sound field into five categories in terms of "Reverberance" [from "much" to "little"], "Spatial impression" [from "large" to "small"], "Home practice (for the usual piano practice at home)" [from "adequate" to "inadequate"], and "Hall practice (for the piano practice for playing in a certain hall)" [from "adequate" to "inadequate"]. This method of evaluating the sound field was used in the former experiments, and it is thought that the results of the experiment were not influenced by the procedure of this method. ${ }^{3)}$

The subjects were four piano teachers, a pianist, and a piano engineer selected from among the subjects used in the previous experiment. ${ }^{2)}$ There were 18 kinds of sound field consisting of the three types of sound absorption ([1]-[3]) mentioned above and six howling margins $(\mathrm{HM}=\infty \mathrm{dB}, 20 \mathrm{~dB}, 15 \mathrm{~dB}, 10$ $\mathrm{dB}, 6 \mathrm{~dB}, 3 \mathrm{~dB}$ ) as the amount of reflective added sounds using electro-acoustic technology. Each subject evaluated a sound field once after repeatedly playing the piano for enough judgment. A howling margin of $3 \mathrm{~dB}$ smaller than $6 \mathrm{~dB}$, minimum value in the previous experiment, was added. Thus, we were also able to evaluate a sound field in which the degree of added reflective sounds had been increased.

\section{RESULTS AND DISCUSSION}

The evaluations for "Reverberance," "Spatial impression," "Home practice," "Hall practice," are shown in Fig. 4. For the purpose of comparison, Fig. 5 shows the results of the previous experiment ${ }^{2)}$ in the same scale of the horizontal axis as Fig. 4.

Figure 4(a) shows the average values of evaluations on "Reverberance" by six of the eleven subjects from the previou experiment and so can be compared with Fig. 5(a).

Figure 4(b) shows the average values of evaluations on "Spatial impression" by five of the original eleven subjects and so can be compared with Fig. 5(b). Figures 4(a) and (b) show that for both "Reverberance" and "Spatial impression," the evaluations of "much" or "large" increased respectively for any type of sound absorption as the added reflective sounds increased. With regard to sound absorption conditions, the difference in the evaluations of conditions [1] and [2] was very little, but the evaluation of condition [3] was a little low compared with the other two conditions. This indicates that the evaluation is influenced by the degree of sound absorption. The comparison of Fig. 4(a) and 5(a) ("Reverberance") to Fig. 4(b) and 5(b) ("Spatial impression") show that the evaluations made by actually playing the piano in this experiment are roughly the same as those made by listening binaurally through headphones in the previous experiment.

Figures 4(c) and (d) show the evaluations on "Home practice." As in the previous experiment, the results of evaluations were devided into two groups of subjects. Figures 4(c) and (d) show the average values of evaluations of four subjects and of two subjects respectively in the same groups as in the previous experiment. Therefore, these can be compared with Fig. 5(c) and (d). The evaluations made by four people for any type of sound absorption are shown in Fig. 4(c). The evaluations of HM conditions $\infty \mathrm{dB}, 20 \mathrm{~dB}$, and $15 \mathrm{~dB}$ which had few or no added reflective sounds were high, and those of $\mathrm{HM}$ conditions $10 \mathrm{~dB}, 6 \mathrm{~dB}$, and $3 \mathrm{~dB}$ were low. Compared to the above results, there are differences in the evaluations made by two subjects shown in Fig. 4(d) due to the sound absorption conditions. Also the evaluations of sound fields with a lot of added reflective sounds were higher 


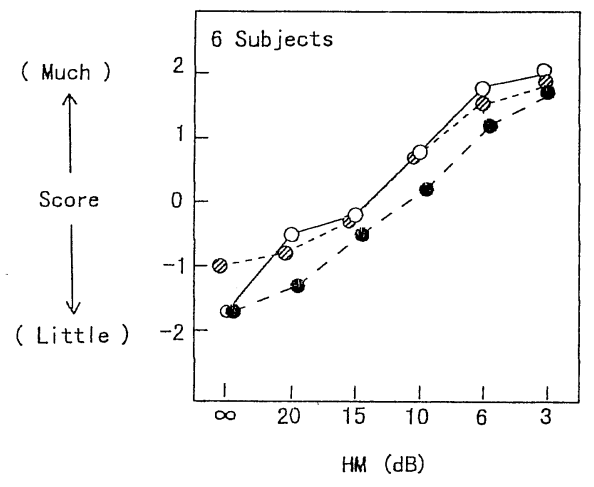

(a) "Reverberance"

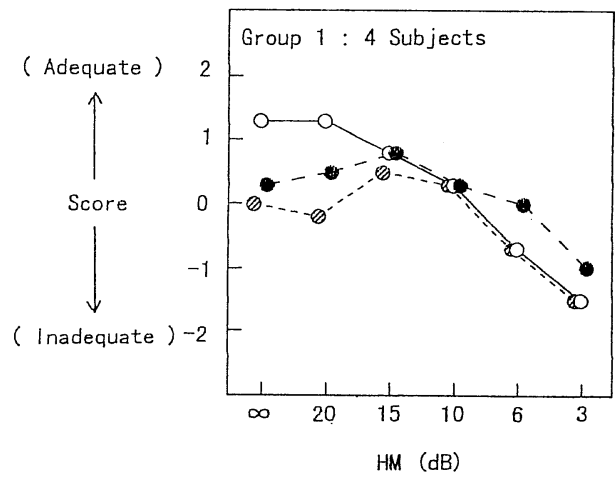

(c) "Home practice"

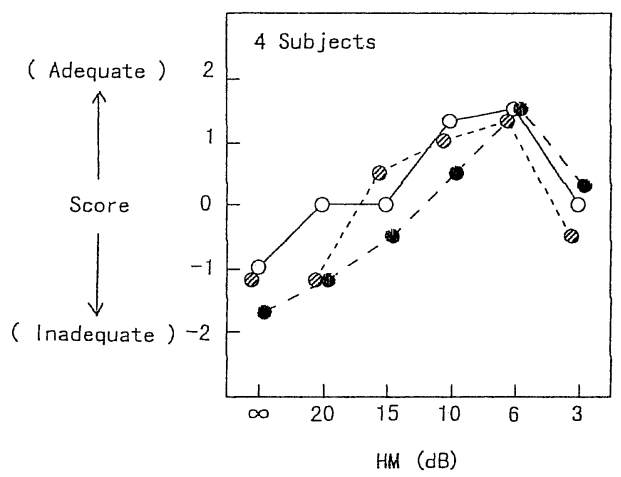

(e) "Hall practice"

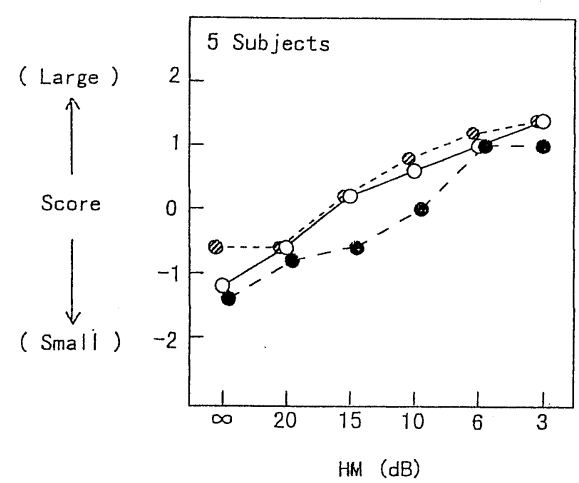

(b) "Spatial impression"

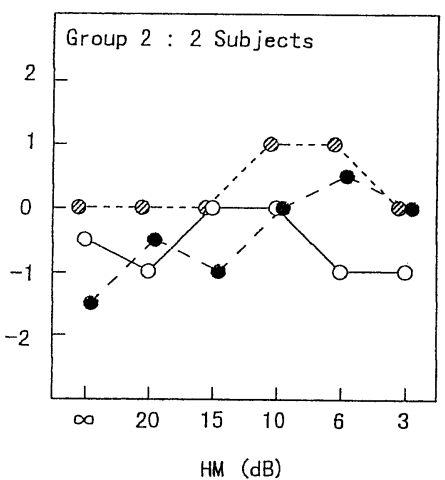

(d) "Home practice"

Fig. 4 Results of the subjective experiments by actually playing the piano (shown by the average score of five categories judgment in the vertical axis, where HM in the horizontal axis is howling margin).

, sound absorption condition [1]; $\bigcirc$, sound absorption condition [2]; •, sound absorption condition [3]. 

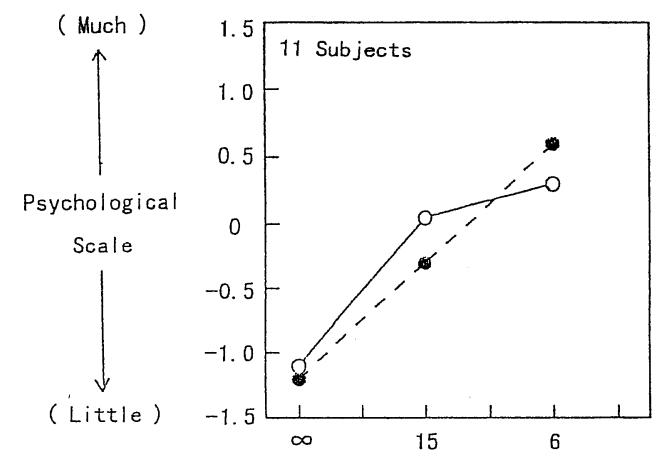

$H M(d B)$

(a) "Reverberance"

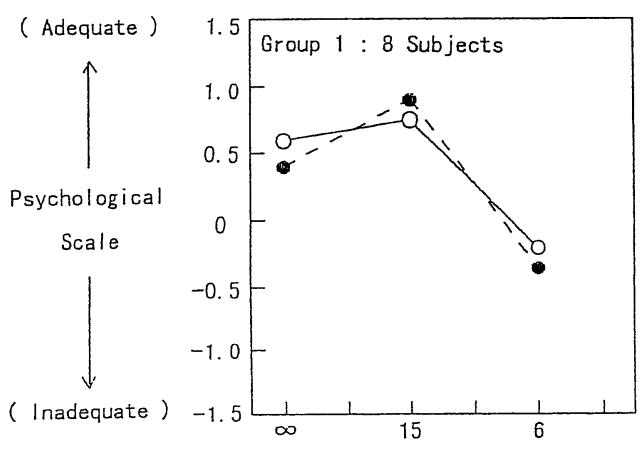

(c) "Home practice"

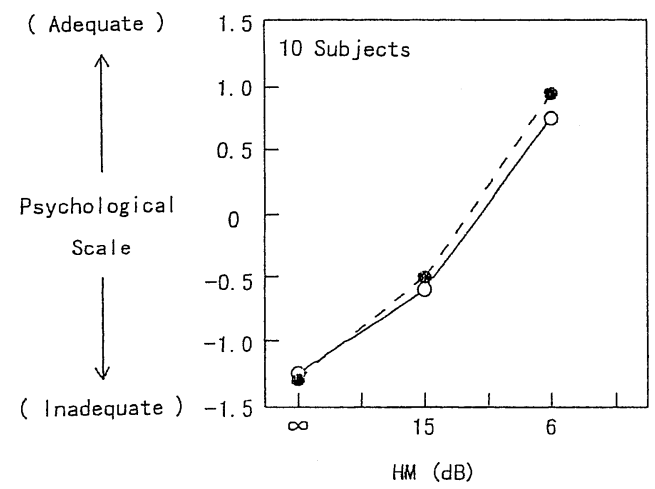

(e) "Hall practice"
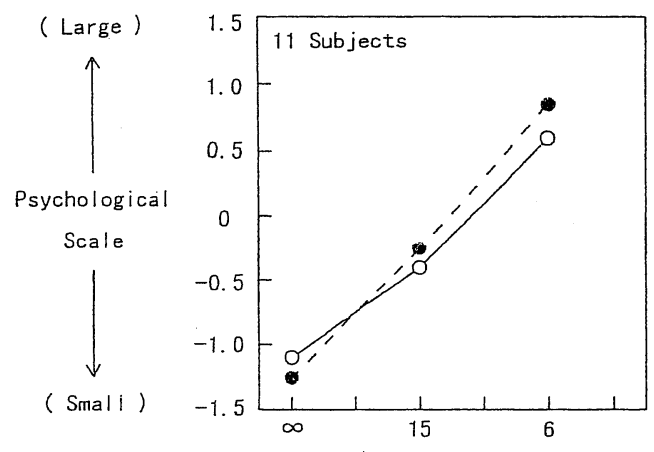

$H M(d B)$

(b) "Spatial impression"

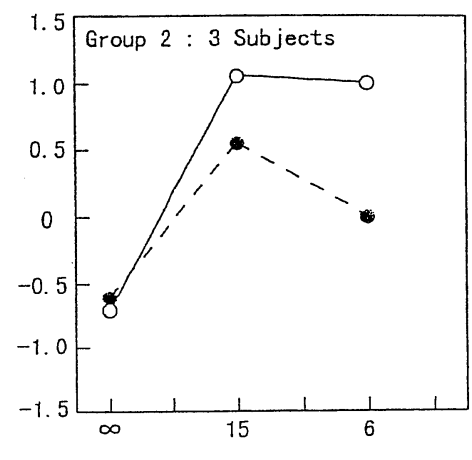

$H M$ (dB)

(d) "Home practice"

Fig. 5 Results of the subjective experiments by listening binaurally through headphones, where the piano sound was recorded by dummy-head (shown by the psychological scales analyzed by the successive categories method in the vertical axis). ${ }^{2)} \bigcirc: \bar{\alpha}$ (average absorption coefficient) $=0.35, \bullet: \bar{\alpha}=0.5$. 
than those with little or no added reflective sounds, but the evaluations of all $\mathrm{HM}=3 \mathrm{~dB}$ conditions were low. With regard to the evaluations of sound absorption conditions, the evaluations made by four subjects of condition [2] with $\mathrm{HM}=\infty \mathrm{dB}$ and 20 $\mathrm{dB}$ were the highest and evaluations of condition [3] were next. The evaluations of condition [1] were the lowest not only in $\mathrm{HM}=\infty \mathrm{dB}$ and $20 \mathrm{~dB}$, but also in $\mathrm{HM}=15 \mathrm{~dB}$ and $10 \mathrm{~dB}$. In other words, these four subjects prefered a "dead" sound field (a sound field with little reverberation) regardless of any additions of reflective sounds. On the other hand, two subjects rated condition [1] highest in almost all HM, and rated conditions [2] and [3] high in $\mathrm{HM}=6 \mathrm{~dB}, 3 \mathrm{~dB}$ but the other evaluations are obscure. In contrast with the other four subjects, they prefered a "live" sound field (a sound field with a lot of reverberation) regardless of any additions of reflective sounds. Comparing these results with those shown in Fig. 5(c) and (d) the evaluations made by four subjects in this experiment when actually playing the piano are roughly equal to the evaluations made when listening binaurally through headphones, but as for the evaluations made by two subjects the distinct relation was not obtained. These two subjects, however, showed the same tendency as those listening binaurally through headphones to evaluate $\mathrm{HM}=\infty \mathrm{dB}$ conditions as not high in general.

Figure 4(e) shows the average values of evaluations on "Hall practice" by four of the ten subjects used in the previous experiment. Thus, this figure can be compared with Fig. 5(e). Figure 4(e) shows that under any sound absorption conditions, the evaluations became higher as more reflective sounds were added, but the evaluations of conditions with $\mathrm{HM}=3 \mathrm{~dB}$ became conversely lower. Each subject commented that the sound field in $\mathrm{HM}=3 \mathrm{~dB}$ was too reverberant compared with an actual hall. With regard to sound absorption conditions, the evaluations of condition [3] were lower than of conditions [1] and [2], but with $\mathrm{HM}=6 \mathrm{~dB}$ the evaluations of the three sound absorption conditions were almost the same and were the highest of any sound absorption conditions. The comparison of Fig. 4(e) and Fig. 5(e) which shows the results of evaluations of "Hall practice" with Fig. 4(a) and Fig. 5(a) which show the evaluations of "Reverberance," and Fig. 4(b) and Fig. 5(b) which show the evaluations of "Spatial impression," show that eval- uations made by actually playing the piano were indicate almost the same as the evaluations made in the previous experiment by listening binaurally through headphones, except that the evaluations of conditions where $\mathrm{HM}=3 \mathrm{~dB}$ were low.

The above-mentioned results are summarized as followings :

(1) The evaluations made by actually playing the piano and those made by, listening binaurally through headphones are roughly equal.

(2) The influence of the degree of sound absorption and the player's preference for reverberance come out more clearly in the evaluations made by actually playing the piano than in those made by listening binaurally through headphones. Especially with regard to the evaluation of "Home practice," the players who prefered a "dead" sound field with little reverberation evaluated a high degree of sound absorption with little added reflective sounds as better. On the other hand, the players who prefered a "live" sound field with a lot of reverberation evaluated a low degree of sound absorption with a lot added reflective sounds as better. This indicates that when actually playing the piano, the player can easily recognize the degree of sound absorption which causes early reflective sounds to influence the live piano sound, and that the player's preference for reverberance influences the evaluation made when the player plays the piano.

\section{CONCLUSION}

Subjective evaluations of a sound field actively controlled by adding reflective sounds in a piano practice room were made. The subjective evaluations made by actually playing the piano and those made in a previous experiment ${ }^{2)}$ by listening binaurally through headphones were compared. The evaluations were almost the same but when actually playing the piano, the piano player's preference for reverberance strongly influenced the evaluations and especially influenced on evaluation of "Home practice." This suggests it may be necessary to take into account the sound absorption of a room as well as the piano player's preference for reverberance in the sound field actively controlled by adding reflective sounds using electro-acoustic technology, and similarly in the sound field controlled by architectural means. $^{3)}$ 


\section{T. FUJITA and S. ANDO : SUBJECTIVE EVALUATION OF SOUND FIELD IN PIANO ROOM}

\section{ACKNOWLEDGEMENTS}

The authors thank Professor Toshio Sone of Tohoku University for his valuable advices and Mr. Hajime Suzuki of Yamaha Corp. for his assistance in the experiment.

\section{REFERENCES}

1) T. Fujita, S. Ando, and K. Yamaguchi, "A study on the active control of sound field for a piano practice room," J. Acoust. Soc. Jpn. (J) 50, 986-996 (1994) (in Japanese).

2) T. Fujita and S. Ando, "A study on the influence of room absorption on the effectiveness of active sound field control in a piano practice room," J. Acoust. Soc. Jpn. (J) 52, 779-787 (1996) (in Japanese).

3) T. Fujita and K. Yamaguchi, "A study on the acoustical characteristics of a piano practice room," Acustica 63, 211-221 (1987).

4) Y. Yamasaki and T. Itow, "Measurement of spatial information in sound fields by closely four point microphone method," J. Acoust. Soc. Jpn. (E) 10, 101-119 (1989).

5) S. Ando, "Sound field control of a small room for music training and its study," Tech. Rep. Music Acoust., MA90-13, Acoust. Soc. Jpn. (1990) (in Japanese).

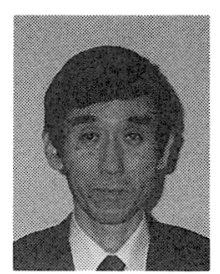

Takashi Fujita was born in Japan on Nov. 13, 1947. He received the B. E. degree in electrical engineering from Tohoku University in 1970. He joined Nippon Gakki Co., Ltd. (the present name is Yamaha Corporation) in 1970 and he was engaged in studies and designs of the architectural acoustics of recording studio and piano practice room and the sound reinforcement system of multi-purpose hall from 1972 to 1998. He received the $\mathrm{Ph}$. D. degree in information sciences from Tohoku University in 1998. Currently he works in the field of the acoustics of electronic musical instruments. He is a member of the Acoustical Society of Japan and the INCE/Japan.

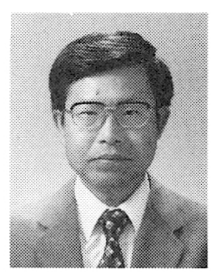

Shigeo Ando was born in Japan on April 24, 1944. He received the B. E. and M. E. degrees in electrical engineering from Gifu University in 1967 and 1969, respectively. In 1969 he joined Nippon Gakki Co., Ltd. (the present name is Yamaha Corporation) and he was engaged in studies of analysis and synthesis of musical instrument tones using digital signal processing from 1972 to 1986 . He received the Dr. Eng. degree from Tohoku University, Japan, in 1989. Since 1981 he has studied and developed the sound field control for use in music practice room using DSP (digital signal sound processor). He is a member of the Acoustical Societv of Japan. 